\title{
Boundary Layer Separation Under Strong Adverse Pressure Gradient Over Smooth and Rough Walls
}

\author{
Pouya Mottaghian, Junlin Yuan and Ugo Piomelli \\ Department of Mechanical and Materials Engineering, Queen's University
}

\section{Synopsis}

Separation of the turbulent boundary layer, is among the most critical phenomena that determine the efficiency of flow devices, ranging from airplane wings to turbine and compressor blades, to curved ducts. In many of these cases roughness is present, and increases the wall friction, due to the contribution of pressure drag. In this study Large-eddy Simulations are carried out on flat-plate boundary layer over smooth and rough surfaces in the presence of an adverse pressure gradient, strong enough to induce separation. Roughness causes early flow separation due to increased momentum deficit and decrease of turbulent kinetic energy production.

\section{Introduction}

We consider the turbulent flow over a flat plate with a suction and blowing velocity profile imposed at the freestream. This velocity profile induces a strong adverse pressure gradient (APG) that causes the flow to separate and then to reattach downstream. This type of pressure-driven separation is relevant to many flows (airfoils, ducts, etc.) and, unlike the case of geometry induced separation (such as that in the backward-facing step), the prediction of both separation and reattachment is challenging. Previous numerical simulations of this flow in the turbulent regime [1] have shown that large coherent structures are generated in the separation zone and lifted upward into the shear layer and over the separated-flow region. Near the reattachment point these structures impinge on the wall and break apart, generating a highly unsteady three-dimensional flow. The effect of roughness is know to alter to some extent these dynamics, but the knowledge of the interaction between APG leading to separation and roughness is limited. Boundary layers subjected to strong APG on a rough wall have been studied experimentally in flows over a hill or a ramp $[2,3]$, where the roughness was shown to cause earlier separation, and a larger separation bub1ble compared to the smooth-wall flow. This has been attributed to a larger mean momentum deficit on the rough wall. However, roughness is also expected to lead to higher turbulence intensities, which should contribute to a delay of the separation. Therefore, the cause of the earlier separation is not yet fully understood. In this study, we carry out large-eddy simulations (LES) of flat plate boundary layers to investigate the physics underlying rough wall flow separations.

\section{Methods and Results}

The simulations are performed using a well-validated code that solves continuity and momentum equations on a staggered grid using second-order, central differences for all terms, and a second-order semi-implicit time advancement [4]. The deceleration is imposed by a spatially varying freestream velocity in the wall-normal direction, $V_{\infty}(x)$ (Fig. 1)); An immersed-boundary method (IBM) based on the volume-of-fluid approach [5] is used to model the roughness as a dense distribution of randomly rotated ellipsoids (sand grains).

A smooth-wall and a rough-wall case are simulated in the same conditions. Grid sizes for the smooth and rough case have, respectively, $2560 \times 384 \times 384$ and $2048 \times 384 \times 384$ grid points. The roughness Reynolds number, $k^{+}=k u_{\tau} / \nu$ (where $u_{\tau}$ is the friction velocity, $\nu$ the kinematic viscosity), is 60 in the upstream equilibrium region.

Figure 2 shows the friction coefficient $C_{f}=2 \tau_{w} / \rho U_{\infty, o}$ (where $U_{\infty, o}$ is the freestream velocity at the inlet) above the roughness sublayer. In the rough-wall case the flow separates earlier than on the smooth wall, and the separation bubble is longer and taller than the smooth case. In the roughness sublayer, APG leads to faster decrease of $\langle\bar{u}\rangle$ (compared to the outer layer) due to larger small-scale separations region downstream of roughness elements which do not emerge from roughness sublayer until $x / \theta_{o}=170$; an increasingly larger percentage of the $x z$ plane is associated with zero or negative $\langle\bar{u}\rangle$, leading to the earlier separation. Note that, inside the roughness layer, the mean velocity becomes negative even earlier.

The mean streamwise-velocity profiles at two locations are shown in (Fig. 3). Roughness leads to lower mean momentum in the boundary layer due to the higher Reynolds shear stress and the increased momentum deficit is the main reason of early separation at rough wall flow.

The APG is found to noticeably decrease the TKE magnitude on the rough wall for $100<x / \theta_{o}<130$; it can be shown that this is due to a significant decrease of TKE production. Furthermore, after separation, the turbulent intensity increases in the shear layer because turbulent eddies detach from the wall and grow significantly inside the separating shear layer; in this process, the structures depend on the outer layer input, i.e., the freestream deceleration and acceleration, instead of that from the wall, i.e., the roughness.

\section{Conclusions}

Large eddy simulations of a separated turbulent boundary layer over a flat plate were performed to study the effects of wall roughness. The rough-wall case has a much larger separation zone with both earlier separation and later reattachment because roughness increases momentum deficit leads to decrease of vertical gradient of streamwise velocity in rough wall flow. In addition, mixing decreases significantly before separation comparing to inlet in rough case and is not strong enough to delay

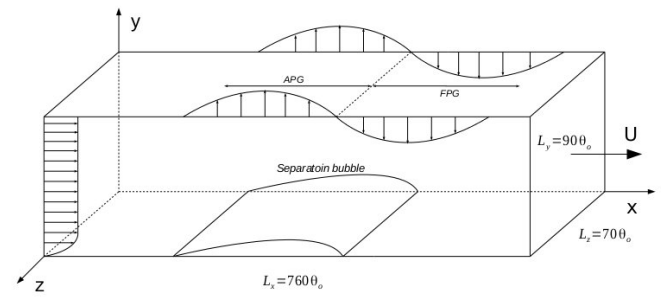

Figure 1: Computational setup

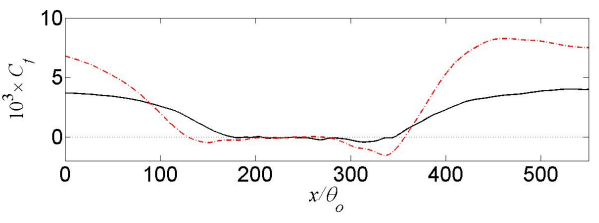

Figure 2: Streamwise development of the friction coefficient.

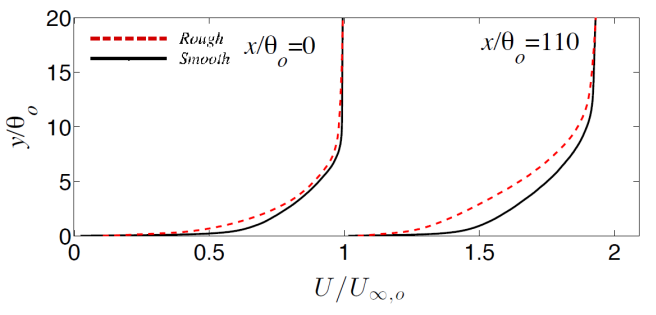

Figure 3: Evolution of the streamwise mean velocity in the upstream equilibrium region $\left(x / \theta_{o}=0\right)$ and immediately before separation $\left(x / \theta_{o}=110\right)$. 


\section{References}

[1] Y. Na and P. Moin, Direct numerical simulation of a separated turbulent boundary layer, J. Fluid Mech., vol. 370, pp. 175-201, 1998.

[2] S. Song, D. B. DeGraaff, and K. Eaton, Experimental studies of a separating, reattaching, and redeveloping flow over a smoothly contoured ramp, Int. J. Heat Fluid Flow, vol. 21, pp. 512-519, 2000.

[3] S. Cao and T. Tamura, Experimental study on roughness effects on turbulent boundary layer flow over a two-dimensional steep hill, Journal of wind engineering, vol. 94, pp. 1-19, 2006.

[4] A. Keating, U. Piomelli, K. Bremhorst, and S. Nešić, Large-eddy simulation of heat transfer downstream of a backward-facing step, J. Turbul., vol. 5, p. N20, 2004.

[5] A. Scotti, Direct numerical simulation of turbulent channel flows with boundary roughened with virtual sandpaper,Phys. Fluids, 18:31701, 2006. 